\title{
Nanostructures Viewed through Low Voltage Electron Microscopy
}

\author{
Daniela R. Radu* and Cheng-Yu Lai, \\ Delaware State University, Dover, DE, USA (dradur@desu.edu)
}

Materials that present nanostructural features, either internally-as in nanoporous structures, or externally_as in nanoparticles with various shapes and aspect ratios, pose a challenge in visualizing their nanoscale features via low-voltage microscopy. The LV EM 5, the only benchtop TEM in the market, offers a variety of tools that overcome the typical low voltage drawbacks. These enhancements will be presented in the context of two projects. The first part of the presentation will discuss the use of LV EM 5 in SEM-mode in a project targeting the application of mesoporous silica nanomaterials in carbon capture, with emphasis on the particles analysis. In the second part, the TEM and SEM modes will be presented in the context of a solar research project, where the solar device is fabricated solely through solution processing and both nanoparticle precursors and films are subjected to microscopy investigation.

Mesoporous Silica. The ability of amine groups to capture $\mathrm{CO}_{2}$ through a simple chemical reaction, prompted the idea of combining the $\mathrm{CO}_{2}$ chemical absorption capability of aminederivatized surfaces with the adsorption capability of a unique folded nanosheet structure and ultra-large surface area of a novel porous silica platform. From economic perspective, the synthetic path of this novel platform involves a low-cost, scalable process, involving inexpensive starting materials and room temperature synthesis.

The synthesis of mesoporous silica nanostructures (MSN) involves a simple condensation reaction of a silica source around a template (surfactant, block copolymer etc.). Various surfactants and pore enhancing molecules have been explored for changing pore size and pore accessibility, while maintaining spherical morphology of the particles. Upon synthesis, the template is removed by calcination or extraction in an acidic solution for cationic surfactants or alcoholic solution for non-ionic surfactants. A survey of various synthetic methodologies has lead to obtaining a unique nanostructure, nanosheet-like silica nanosphere (NSN).

Nanoprecursor-Based Solar Cells. Chalcogenide semiconductors, such as copper indium gallium diselenide - $\mathrm{Cu}(\mathrm{In}, \mathrm{Ga}) \mathrm{Se}_{2}$ (CIGS), kesterites - $\mathrm{Cu}_{2} \mathrm{ZnSn}(\mathrm{S}, \mathrm{Se})_{4}$, (CZTS), and recently proposed iron chalcogenide, $\mathrm{Fe}_{2} \mathrm{SiS}_{4}$ and $\mathrm{Fe}_{2} \mathrm{GeS}_{4}$, offer bandgaps close to ideal for the absorber material in thin-film photovoltaic (PV) technologies. Progress is continuously made in this arena both in terms of small cell efficiencies and in terms of larger area module efficiencies. Besides the cost aspect on Earth applications, chalcogenide solar cells are also interesting for space applications because of their excellent stability against particle irradiation and development of high efficiency flexible cells. Roll-to-roll printed nanoparticle technologies for flexible solar cells could offer the combination of fast, atmospheric pressure deposition, a lightweight substrate, and a thin, inexpensive absorber layer, each of which decreases the cost and the weight of the final solar cell or module, toward making solar energy affordable. Nanoparticles and nanoparticles-originated polycrystalline layers of $\mathrm{Cu}_{2} \mathrm{ZnSn}(\mathrm{S}, \mathrm{Se})_{4}$, (CZTS), and $\mathrm{Fe}_{2} \mathrm{SiS}_{4}$ and $\mathrm{Fe}_{2} \mathrm{GeS}_{4}$ from nanoparticles synthesis to the solar cell performance will be reviewed. ${ }^{1-3}$

NSN Materials. The synthetic routes reported for porous silica nanospheres obtained with the co-solvent method have employed energy intensive reaction conditions and used semi-scalable 
methods such as microwave-assisted synthesis. ${ }^{4}$ The materials synthesized with our conditions require room temperature, in air. Characterization of materials via nitrogen porosity indicates a very large surface area and pore volume (Table 1).

Table 1. BET Surface area of synthesized materials

\begin{tabular}{|c|c|c|c|}
\hline Material & NSN-1 & NSN-2 & NSN-3 \\
\hline BET Surface area (m $\left.\mathbf{~ m}^{\mathbf{2}} \mathbf{g}\right)$ & 1070 & 1240 & 1398 \\
\hline Co-solvent(s) & Ethyl ether & $\begin{array}{c}\text { Ethyl ether- } \\
\text { ethanol }\end{array}$ & Ethyl ether \\
\hline
\end{tabular}

A comparison of images obtained via SEM and LV SEM show the capability of LV EM 5 to visualize nano-features close in nm range.

Nanoprecursor-Based Solar Cells. Nanoparticles structure and size as well as surface properties along with nanoparticle coatings and film thermal treatment, all play an immense role in making a continuous, crack-free, crystalline absorber layer. The instrument facile operation enables analysis of a large amount of samples on daily-basis, which is beneficial for synthetic optimization and fast advancement of research. Furthermore, identification of nanoparticle composition when particle size is below $100 \mathrm{~nm}$ becomes cumbersome with XRD due to line broadening in XRD. Investigation of NPs via TEM imaging and electron diffraction patterns highlight the potential of LV microscopy in such research.

The LV EM 5 instrument in SEM mode enables early identification of both physical (impurity) and mechanical (cracks) defects in sample surface and cross section to identify showstoppers before transferring the film to the next step in the device fabrication flow.

Both projects benefit from the easy access of the LV EM 5. In contrast to high-voltage SEM and TEM counterparts, the LV enables high-throughput analysis. While not being able to substitute for high-voltage microscopy, the bench top LV EM 5 is a versatile, inexpensive and easy to operate system. The instrument represents an excellent educational tool that could be solely operated by students/researchers without the need of a specialized operator.

Acknowledgements. The authors thank DOD (Award W911NF-14-1-0071) for funding support in acquiring the LV EM 5.

\section{References}

1. Cao, Y.; Denny, M. S.; Caspar, J. V.; Farneth, W. E.; Guo, Q.; Ionkin, A. S.; Johnson, L. K.; Lu, M.; Malajovich, I.; Radu, D.; Rosenfeld, H. D.; Choudhury, K. R.; Wu, W., HighEfficiency Solution-Processed Cu2ZnSn(S,Se)4 Thin-Film Solar Cells Prepared from Binary and Ternary Nanoparticles. Journal of the American Chemical Society 2012, 134, 15644-15647.

2. $\quad$ Radu, D. R.; Caspar, J. V.; Johnson, L. K.; Rosenfeld, H. D.; Malajovich, I.; Lu, M. Copper zinc tin chalcogenide nanoparticles. WO2010135622A1, 2010.

3. $\quad$ Radu, D. R.; Caspar, J. V.; Lu, M.; Johnson, L. K.; Cao, Y.; Ionkin, A. S.; Malajovich, I.; Rosenfeld, H. D.; Sun, F.; Tassi, N. G., CZTS absorber layer in thin film p-n junction solar devices from quaternary nanoparticle precursors. Prepr. Symp. - Am. Chem. Soc., Div. Fuel Chem. 2011, 56, 179. 